\title{
Procalcitonin-guided algorithms of antibiotic therapy in the intensive care unit with sepsis: a meta-analysis
}

\author{
Yuhan ZHU' ${ }^{1}$, Xiaoxu SHEN²*, Yongyao WEN ${ }^{3}$, Zhen WANG ${ }^{1}$, Zhaobo WANG ${ }^{1}$, Xin DONG ${ }^{1}$, Jiebai LI ${ }^{1}$, Qingqing HAN ${ }^{1}$, Jing ZHAO ${ }^{1}$ \\ and Lei LIU' ${ }^{1}$ \\ ${ }^{1}$ Dongzhimen Hospital Affiliated to Beijing University of Chinese Medicine, Beijing 100029, China \\ ${ }^{2}$ Cardiovascular Department of Dongzhimen Hospital Affiliated to Beijing University of Chinese Medicine, Beijing 100700, China \\ ${ }^{3}$ Guangxi University of Chinese Medicine, Nanning 530001, China
}

\begin{abstract}
Objective: To systematically review the effectiveness and safety of antibiotic therapy in sepsis under the guidance of procalcitonin in ICU.

Data sources: PubMed, The Cochrane Library (through October 2016), EMbase, Web of Science, reference lists of retrieved publications. No limitations regarding the language of publications.

Study selection: We included only randomized controlled studies reporting on critically ill patients with sepsis managed with a PCT-guided algorithm vs. Routine practice.

Data extraction: Data on study characteristics, interventions, and outcomes were retrieved by two independent reviewers. Risk ratios, mean differences, and 95\% confidence intervals were calculated by implementing both the Mantel-Haenszel fixed effect model and the Mantel-Haenszel random effect model.

Data synthesis: Thirteen randomized controlled studies involving 4728 ICU septic patients were included. Compared with the routine particle, PCT-guided algorithms decreased the total duration of antibiotic therapy (REM; $\mathrm{MD}=-1.60 ; 95 \% \mathrm{CI},-2.14$ to $-1.06 ; \mathrm{Z}=5.79 ; P<0.00001$ ), and the 28-day mortality had also been reduced ( $(\mathrm{FEM} ; \mathrm{MD}=0.86 ; 95 \% \mathrm{CI}, 0.76$ to $0.97 ; \mathrm{Z}=2.39 ; P=0.02$ ). However, between the PCT-guided group and the routine practice group, the length of ICU and hospital stay, the ICU mortality, in hospital mortality and the rates of clinical cure had no significant difference.

Conclusions: The PCT-guided algorithms could decrease the total duration of antibiotic therapy and reduce the 28-day mortality of septic patients in ICU without improving the length of ICU and hospital stay, the ICU mortality, in hospital mortality and the rates of clinical cure. Further research is still necessary before the wide adoption of this strategy.
\end{abstract}

\section{Introduction}

Sepsis is defined as a systemic inflammatory response syndrome (SIRS) plus documented or suspected infection [1], associated with increasing hospitalization and mortality [2,3]. Antibiotic therapy is the key to cure and reduce the mortality of sepsis. Procalcitonin(PCT), a calcitonin precursor, is a promising biomarker to diagnose the potential sepsis [4]. Even though, Procalcitonin-guided algorithms of antibiotic therapy in intensive care unit is still not universally accepted [5]. Researchers have found that PCT-guided algorithm may reduce antibiotic exposure of sepsis in ICU, but cannot reduce the patients' mortality [6,7]. It remains unclear if the Procalcitonin-guided algorithmic can improve health outcomes compared with routine practice in ICU. Therefore, we performed the Meta-analysis of the randomized controlled trials reporting on the outcomes of septic patients in ICU.

\section{Materials and methods}

\section{Data sources and searches}

We systematically searched MEDLINE, the Cochrane Library, EMbase and Web of Science (from inception up to October 31,2016) to identify relevant randomized controlled trials(RCTs) by using the search terms: procalcitonin AND sepsis AND intensive care unit AND (antibiotic therapy OR Anti-Bacterial Agents OR Anti-Infective) AND Random. The reference lists of the initially retrieved articles were also reviewed. If necessary, the corresponding authors of each one of the included studies were contacted by e-mail for additional information.

\section{Selection of studies}

Two investigators independently carried out the computerized literature search and local the potentially eligible articles. The inclusion criteria in this meta-analysis is that critically ill patients with sepsis managed with a PCT-guided algorithm vs. Routine practice. No limitations regarding the country, time or language of publications. We excluded trials performed outside the intensive care unit(ICU).

\section{Outcome measures}

The duration of antibiotic therapy, length of ICU and hospital stay, 28-day all-cause mortality, ICU and in hospital mortality, the rates of clinical cure.

Correspondence to: Xiaoxu Shen, Cardiovascular Department of Dongzhimen Hospital Affiliated to Beijing University of Chinese Medicine, Beijing 100700, China, Tel: 18518185147; Fax: 64287519; E-mail address: alienstg@163.com

Key words: procalcitonin, sepsis, intensive care unit, meta-analysis

Received: November 24, 2017; Accepted: January 15, 2018; Published: January 18,2018 


\section{Data extraction and quality assessment}

Two investigators dependently reviewed the full text of the retrieved articles and reported the results in a structured dataset. Disparities between investigators regarding the inclusion of each trial were resolved by consensus by a third independent investigator. The data's included first author, year and country of publication, ICU type, study design, number of patients managed with Procalcitonin-guided algorithmic vs. routine practice, details of the PCT-implemented algorithm. The assessment of the methodologic quality of the included RCTs were followed the recommendations exemplified in the Cochrane handbook for systematic reviews of interventions and summarized in a domain-based evaluation of the following components: random sequence generation, allocation concealment, blinding, incomplete outcome data, selective reporting and other bias [8].

\section{Data synthesis and analysis}

We used Review Manager 5.3 to conduct the statistical analysis. The between-study heterogeneity was assessed by the chi-squared test and its extent was quantified by the $I^{2}$ statistic $\left(I^{2}\right.$ values of $25 \%, 50 \%$, and $75 \%$ were considered to represent low, moderate and severe statistical inconsistency [9]. If significant heterogeneity could be detected, subgroup analysis and sensitivity analysis will be estimated. Continuous outcomes were analyzed using mean differences(MD) and $95 \%$ confidence intervals(CIs). Risk ratio(RR) and 95\% CI were calculated by implementing the Mantel-Haenszel fixed effect model(FE) and the
Mantel-Haenszel random effect model(RE). A $p<0.05$ was thought to indicate statistical significance in this meta-analysis.

\section{Results}

\section{Study selection}

Of the 829 citations found in MEDLINE, the Cochrane Library, EMbase and Web of Science. The majority were excluded for reasons presented in (Figure 1). Thus, thirteen RCTs [10-22] met the inclusion criteria for this Meta-analysis.

\section{Study description}

In Table 1, we list the main characteristics of the included studies that meet our eligibility criteria. All studies [10-22] were published from 2007 to 2016. Their individual sample size ranged from 27 to 1546 patients. Eight single-center studies and Five multi-center studies. All studies had been conducted in ten different countries: Czech Republic, Switzerland, Germany, France, Belgium, China, Brazil, Iran, Australian, Netherlands. Twelve studies were published in the English language and one study was published in the Chinese language. Eleven studies included medical, surgical, and mixed ICUs [10-13,15-19,2122], two focused on surgical ICUs [14,20].

Data regarding the impact of PCT-guided algorithms on the antibiotic therapy are presented in [Table 2]. Data on the quality of the included studies are presented in Table 3. Eight studies are considered

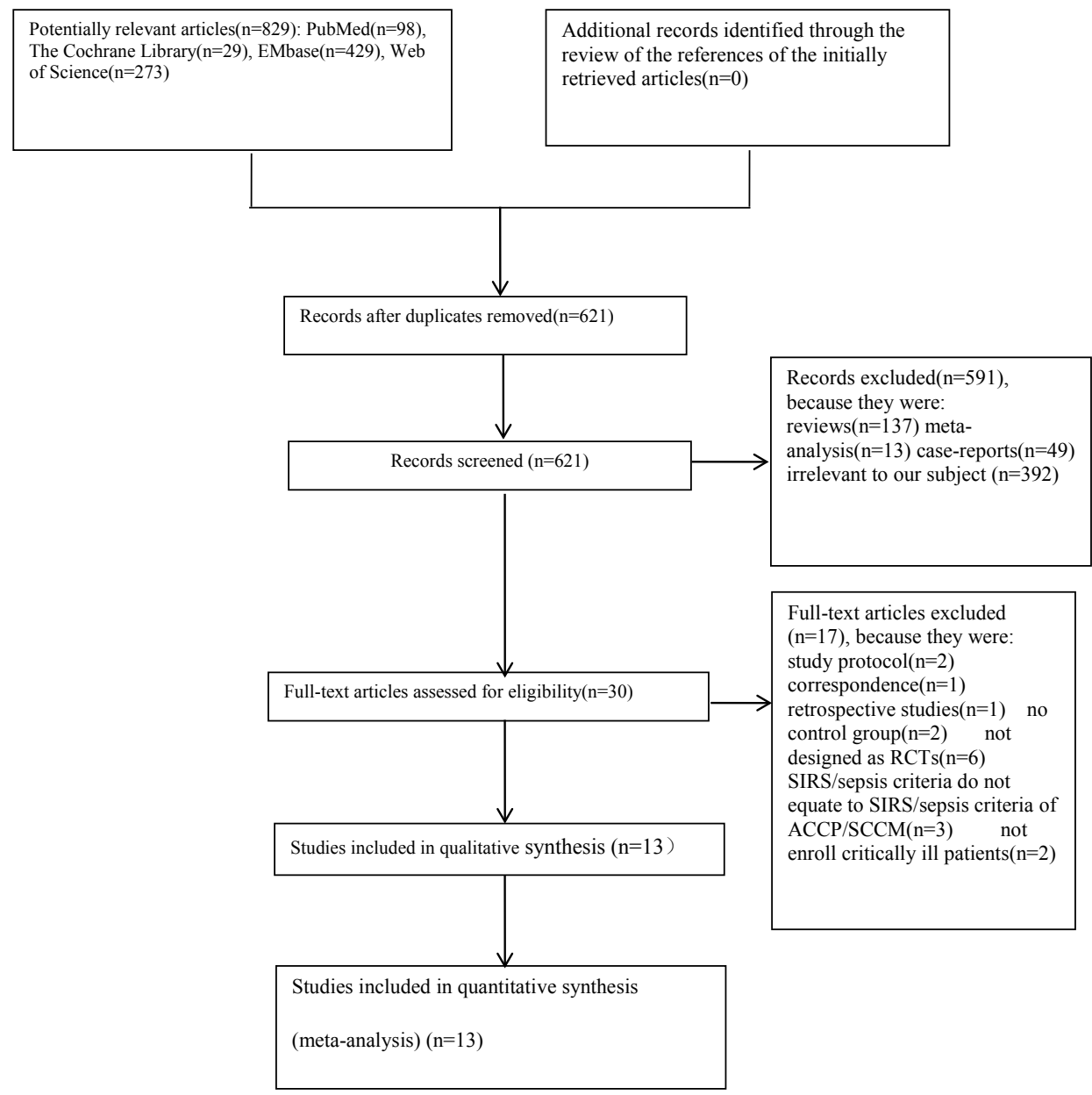

Figure 1. Flow diagram of the literature search and selection process of the studies. 
Table 1. Main characteristics of the studies included in the Meta-analysis: PCT-group vs. routine practice

\begin{tabular}{|c|c|c|c|c|c|c|}
\hline $\begin{array}{l}\text { First Author/Year of } \\
\text { publication (Reference) }\end{array}$ & Svoboda/2007 [22] & $\begin{array}{l}\text { Nobre/2008 } \\
{[19]}\end{array}$ & $\begin{array}{l}\text { Schroeder/2009 } \\
{[20]}\end{array}$ & $\begin{array}{l}\text { Hochreiter/2009 } \\
{[14]}\end{array}$ & $\begin{array}{l}\text { Bouadma/2010 } \\
{[12]}\end{array}$ & $\begin{array}{l}\text { Layios/2012 } \\
{[16]}\end{array}$ \\
\hline Country/Type of ICU & $\begin{array}{l}\text { Czech Republic/4 ICUs } \\
\text { in the same hospital }\end{array}$ & $\begin{array}{l}\text { Switzerland/I ICU in } \\
\text { university hospital }\end{array}$ & Germany/1 surgical ICU & Germany/1 surgical ICU & $\begin{array}{l}\text { France/7 (5 medical,2 } \\
\text { surgical) ICUs in } 5 \\
\text { university-affiliated } \\
\text { hospitals, and } 1 \\
\text { medicosurgical ICU in a } \\
\text { general hospital. }\end{array}$ & $\begin{array}{l}\text { Belgium/5 ICUs of the } \\
\text { univeristy hospital }\end{array}$ \\
\hline Study Design & P,PG,OL,SC & $\mathrm{P}, \mathrm{PG}, \mathrm{OL}, \mathrm{SC}$ & P,PG,OL,SC & P,PG,OL,SC & P,PG,OL,MC & P,PG,SB,SC \\
\hline Inclusion Criteria & $\begin{array}{l}\text { All ICU patients } 18 \text { years } \\
\text { or older who developed } \\
\text { severe sepsis }\end{array}$ & $\begin{array}{l}\text { All ICU patients with } \\
\text { suspected severe sepsis } \\
\text { or septic shock }\end{array}$ & $\begin{array}{l}\text { All ICU Patients after } \\
\text { abdominal surgery and } \\
\text { after start of antibiotic } \\
\text { treatment with the } \\
\text { diagnosis of severe sepsis }\end{array}$ & $\begin{array}{l}\text { All ICU patients } \\
\text { requiring antibiotic } \\
\text { therapy based on } \\
\text { confirmed or highly } \\
\text { suspected bacterial } \\
\text { infections and at least } \\
\text { two concomitant } \\
\text { systemic inflammatory } \\
\text { response syndrome } \\
\text { criteria }\end{array}$ & $\begin{array}{l}\text { All ICU adult patients } \\
\text { with suspected infections } \\
\text { if they were not receiving } \\
\text { antibiotics before } \\
\text { inclusion in the study } \\
\text { or if they had received } \\
\text { antibiotics for less than } \\
24 \mathrm{~h} \text {. }\end{array}$ & $\begin{array}{l}\text { All ICU adult patients } \\
\text { hospitalized for }>48 \\
\text { hrs and suspected of } \\
\text { developing infection } \\
\text { either on admission or } \\
\text { during ICU stay. }\end{array}$ \\
\hline Exclusion Criteria & $\begin{array}{l}\text { chemical or burn trauma, } \\
\text { moribund patients, and } \\
\text { patients who have been } \\
\text { designated as "not full } \\
\text { support" or "do not } \\
\text { resuscitate" }\end{array}$ & $\begin{array}{l}\text { Infections caused by } \\
\text { Pseudomonas aeruginosa, } \\
\text { Acinetobacter } \\
\text { baumanni, Listeria spp., } \\
\text { Legionella pneumophila, } \\
\text { Pneumocystis jiroveci, } \\
\text { or Mycobacterium } \\
\text { tuberculosis, viruses or } \\
\text { parasites or infectious } \\
\text { conditions requiring } \\
\text { prolonged ABx; } \\
\text { ABx started }>48 \text { hrs } \\
\text { before enrollment; } \\
\text { chronic, localized } \\
\text { infections; severely } \\
\text { immunocompromised } \\
\text { patients; withholding } \\
\text { of life support; or } \\
\text { absence of antimicrobial } \\
\text { treatment despite clinical } \\
\text { suspicion of sepsis. }\end{array}$ & $\begin{array}{l}\text { refused informed } \\
\text { consent, or already } \\
\text { had received antibiotic } \\
\text { treatment prior to } \\
\text { admission to the ICU. }\end{array}$ & $\begin{array}{l}\text { Patients who refused } \\
\text { study consent, whose } \\
\text { antibiotic treatment had } \\
\text { been initiated before } \\
\text { intensive care admission, } \\
\text { or who had therapy } \\
\text { limitations }\end{array}$ & $\begin{array}{l}\text { age under } 18 \text { years; } \\
\text { known pregnancy; } \\
\text { expected stay in the } \\
\text { ICU of less than } 3 \text { days; } \\
\text { bone-marrow transplant } \\
\text { or chemotherapy induced } \\
\text { neutropenia; infections } \\
\text { for which long-term } \\
\text { antibiotic treatment is } \\
\text { strongly recommended } \\
\square \text { poor chance of } \\
\text { survival, defined as } \\
\text { a simplified acute } \\
\text { physiology score (SAPS } \\
\text { II) of more than } 65 \text { points } \\
\text { at screening; and do not- } \\
\text { resuscitate orders. }\end{array}$ & $\begin{array}{l}\text { no informed consent, } \\
\text { stay less than } 48 \text { hours }\end{array}$ \\
\hline $\begin{array}{l}\text { Description of PCT- } \\
\text { Guided Algorithms }\end{array}$ & $\begin{array}{l}\text { ABx were encouraged } \\
\text { when severe sepsis with } \\
\text { PCT value }>2 \mu \mathrm{g} / \mathrm{L} \text {, } \\
\text { severe sepsis with PCT } \\
\text { value } \leq 2 \mu \mathrm{g} / \mathrm{L} \text { prompted } \\
\text { to ultrasonography and/ } \\
\text { or CT }\end{array}$ & $\begin{array}{l}\text { ABx were stopped } \\
\text { when PCT levels had } \\
\text { decreased }>90 \% \text { from } \\
\text { the initial value but not } \\
\text { before Day } 3 \text { (if baseline } \\
\text { PCT }<1 \mu \mathrm{g} / \mathrm{L} \text { ) or Day } 5 \text { (if } \\
\text { baseline PCT }>1 \mu \mathrm{g} / \mathrm{L} \text { ). }\end{array}$ & $\begin{array}{l}\text { ABx were discontinued if } \\
\text { clinical signs of infection } \\
\text { improved and the PCT } \\
\text { value was either }<1 \mu \mathrm{g} / \mathrm{L} 1 \\
\text { or decreased to }<35 \% \text { of } \\
\text { the initial concentration } \\
\text { within three consecutive } \\
\text { days }\end{array}$ & $\begin{array}{l}\text { ABx were discontinued } \\
\text { if clinical signs and } \\
\text { symptoms of infection } \\
\text { improved and PCT } \\
\text { decreased to }<1 \mu \mathrm{g} / \mathrm{L} \text { or } \\
\text { the PCT }>1 \mu \mathrm{g} / \mathrm{L} \text { but had } \\
\text { dropped to } 25 \text { to } 35 \% \text { of } \\
\text { the initial value over } \\
\text { three days. }\end{array}$ & $\begin{array}{l}\text { ABx were discontinued } \\
\text { when } \mathrm{PCT}<80 \% \text { of the } \\
\text { peak concentration or an } \\
\text { absolute value }<0.5 \mu \mathrm{g} / \mathrm{L}\end{array}$ & $\begin{array}{l}\text { ABx were discouraged if } \\
\mathrm{PCT}<0.25 \mu \mathrm{g} / \mathrm{L} \text { or } \\
0.50 \mu \mathrm{g} / \mathrm{L} \text {, and more or } \\
\text { less recommended } \\
\text { if } \mathrm{PCT}>1 \mu \mathrm{g} / \mathrm{L} \text { or } 0.50 \\
\mu \mathrm{g} / \mathrm{L}\end{array}$ \\
\hline $\begin{array}{l}\text { Description of Routine } \\
\text { practice }\end{array}$ & $\begin{array}{l}\text { ABx were based } \\
\text { on consultant } \\
\text { surgeon according to } \\
\text { contemporary treatment } \\
\text { protocol of their institute }\end{array}$ & $\begin{array}{l}\text { ABx were decided by } \\
\text { the clinicians based on } \\
\text { empirical rules. }\end{array}$ & $\begin{array}{l}\text { ABx were directed } \\
\text { by clinical signs and } \\
\text { empirical rules. }\end{array}$ & $\begin{array}{l}\text { ABx were applied as } \\
\text { standard regimen over } \\
\text { eight days. }\end{array}$ & $\begin{array}{l}\text { ABx were based on } \\
\text { international } \\
\text { and local guidelines. } \\
\text { However, investigators } \\
\text { were free to decide the } \\
\text { optimum duration of } \\
\text { antibiotic treatment based } \\
\text { on their own assessment } \\
\text { of the infection's clinical } \\
\text { course. }\end{array}$ & $\begin{array}{l}\text { ABx were based on } \\
\text { the physician own } \\
\text { assessment }\end{array}$ \\
\hline $\begin{array}{l}\text { Number of Patients, CT- } \\
\text { group/Control-group }\end{array}$ & $38 / 34$ & $31 / 37$ & $14 / 13$ & $57 / 53$ & $307 / 314$ & $258 / 251$ \\
\hline
\end{tabular}




\begin{tabular}{|c|c|c|c|c|c|c|}
\hline $\begin{array}{l}\text { Liu BH/2013 } \\
{[17]}\end{array}$ & $\begin{array}{l}\text { Deliberato/2013 } \\
\text { [13] }\end{array}$ & $\begin{array}{l}\text { Annane/2013 } \\
{[10]}\end{array}$ & $\begin{array}{l}\text { Najafi/2015 } \\
{[18]}\end{array}$ & $\begin{array}{l}\text { Shehabi/2014 } \\
{[21]}\end{array}$ & $\begin{array}{l}\text { Jong/2016 } \\
{[15]}\end{array}$ & $\begin{array}{l}\text { Bloos/2016 } \\
{[11]}\end{array}$ \\
\hline China/1 ICU & $\begin{array}{l}\text { Brazil/1 ICU of a tertiary } \\
\text { care,private hospital }\end{array}$ & France/8 ICUs & Iran/1 mixed ICU & Australian/11 ICUs & $\begin{array}{l}\text { Netherlands } / 15(3 \\
\text { university medical } \\
\text { centres, } 12 \text { teaching } \\
\text { hospitals) ICUs }\end{array}$ & $\begin{array}{l}\text { Germany/33 } \\
\text { multidisciplinary ICUs }\end{array}$ \\
\hline P, PG,OL,SC & $\mathrm{P}, \mathrm{PG}, \mathrm{SB}, \mathrm{SC}$ & P, PG,SB,MC & P, PG, SB,SC & P, PG, SB,MC & P, PG, OL,MC & P, PG, OL,MC \\
\hline $\begin{array}{l}\text { All ICU patients aged } \\
\text { at least } 18 \text { years and } \\
\text { suspected of developing } \\
\text { infection }\end{array}$ & $\begin{array}{l}\text { All ICU Patients with } \\
\text { microbiologically } \\
\text { confirmed infections with } \\
\text { sepsis, severe sepsis, and } \\
\text { septic shock }\end{array}$ & $\begin{array}{l}\text { All ICU adult patients } \\
\text { with the systemic } \\
\text { inflammatory response } \\
\text { syndrome, acute } \\
\text { dysfunction of at least } \\
\text { one organ, absence of } \\
\text { indisputable clinical } \\
\text { infection and negative } \\
\text { microbial cultures }\end{array}$ & $\begin{array}{l}\text { All ICU Patients with } \\
\text { at least two of the four } \\
\text { criteria including body } \\
\text { temperature above } 38 \square \\
\text { or below } 36 \square, \\
\text { tachycardia }>90 / \text { min, } \\
\text { tachypnea }>20 / \text { min and } \\
\text { leucocytosis }>12 \times 10^{9} / \mathrm{L} \\
\text { or a leftward shift with } \\
\text { more than } 10 \% \text { band } \\
\text { cells or leukopenia } \\
<4 \times 10^{9} / \mathrm{L} \text { were } \\
\text { defined as subject with } \\
\text { SIRS }\end{array}$ & $\begin{array}{l}\text { All ICU Patients older } \\
\text { than } 18 \text { years of age, } \\
\text { admitted to ICU within } \\
\text { the previous } 72 \text { hours, } \\
\text { receiving parenteral and/ } \\
\text { or enteral antibiotics for } \\
\text { a suspected bacterial } \\
\text { infection and expected } \\
\text { to remain in the ICU for } \\
\text { longer than } 24 \text { hours }\end{array}$ & $\begin{array}{l}\text { All ICU patients aged } \\
\text { at least } 18 \text { years, and } \\
\text { received their first } \\
\text { dose of } \\
\text { antibiotics no longer than } \\
24 \text { h before inclusion in } \\
\text { the study }\end{array}$ & $\begin{array}{l}\text { All ICU adult patients } \\
\text { with sepsis or septic } \\
\text { shock }\end{array}$ \\
\hline $\begin{array}{l}\text { onset of antibiotic } \\
\text { therapy more } \\
\text { than } 48 \text { hours before the } \\
\text { date when the cultures } \\
\text { were performed; severe } \\
\text { infection caused by } \\
\text { viruses, Acinetobacter } \\
\text { baumannii or } \\
\text { mycobacteria;chronic } \\
\text { localized infections,and } \\
\text { refused study consent }\end{array}$ & $\begin{array}{l}\text { onset of antibiotic } \\
\text { therapy more } \\
\text { than } 48 \text { hours before } \\
\text { the date when the } \\
\text { cultures were performed; } \\
\text { patients under } 18 \\
\text { years old; known } \\
\text { pregnancy; infections } \\
\text { requiring prolonged } \\
\text { antibiotic therapy, and } \\
\text { osteomyelitis; severe } \\
\text { infection caused by } \\
\text { viruses, parasites, fungi, } \\
\text { or mycobacteria; chronic } \\
\text { localized infections, } \\
\text { and negative cultures in } \\
\text { patients with suspected } \\
\text { sepsis, severe sepsis, or } \\
\text { septic shock. }\end{array}$ & $\begin{array}{l}\text { pregnancy, burns over } \\
\geq 15 \% \text { of body surface } \\
\text { area, trauma, outpatient } \\
\text { or inpatient cardiac } \\
\text { arrest, postorthopaedic } \\
\text { surgery status, drug- } \\
\text { related neutropenia, } \\
\text { withdrawal of life- } \\
\text { supportive therapies or } \\
\text { a decision to withhold } \\
\text { them, indisputable } \\
\text { clinical infection or } \\
\text { antibiotic exposure } \geq 48 \\
\text { h during the time shortly } \\
\text { before ICU admission. }\end{array}$ & $\begin{array}{l}\text { documented infection, } \\
\text { pus from wound or } \\
\text { abscess, empyema, } \\
\text { thrombophlebitis, } \\
\text { infection due to viral or } \\
\text { parasites, hypoxemia } \\
\left(\mathrm{PO}_{2}<60 \mathrm{mmHg} \text { ), }\right. \\
\text { oliguria, Glasgow coma } \\
\text { scale (GCS) } 3 \text { without } \\
\text { sedation, parenteral } \\
\text { antibiotic usage } 24 \\
\text { hours before admission } \\
\text { to ICU, hospitalization } \\
48 \text { hours before } \\
\text { enrollment, conditions } \\
\text { requiring prolonged } \\
\text { antibiotic therapy such } \\
\text { as endocarditis, chronic } \\
\text { localized infection } \\
\text { such as osteomyelitis } \\
\text { and severely } \\
\text { immunocompromised } \\
\text { patients. }\end{array}$ & $\begin{array}{l}\text { patients receiving } \\
\text { antibiotics for surgical } \\
\text { prophylaxis or with } \\
\text { proven bacterial infection } \\
\text { requiring more than } 3 \\
\text { weeks' antibiotic therapy, } \\
\text { isolated systemic } \\
\text { fungal or systemic viral } \\
\text { infection in the absence } \\
\text { of bacterial infection, } \\
\text { neutropenia with a } \\
\text { neutrophil count less than } \\
1,000 \text { cells/ml, receiving } \\
\text { immunosuppressive } \\
\text { agents, cardiac surgery } \\
\text { or trauma or heat } \\
\text { stroke within } 48 \text { hours, } \\
\text { medullary thyroid or } \\
\text { small cell lung cancer, } \\
\text { subject not expected } \\
\text { to survive to hospital } \\
\text { discharge, or known } \\
\text { regnancy }\end{array}$ & $\begin{array}{l}\text { in cases of systemic } \\
\text { antibiotics as } \\
\text { prophylaxis only, } \\
\text { antibiotics solely } \\
\text { as part of selective } \\
\text { decontamination of } \\
\text { the digestive tract, } \\
\text { prolonged therapy } \\
\text { (eg, endocarditis), } \\
\text { expected ICU stay of } \\
\text { less than } 24 \text { h, severe } \\
\text { immuno suppression, } \\
\text { severe infections (due } \\
\text { to viruses, parasites,or } \\
\text { Mycobacterium } \\
\text { tuberculosis),and } \\
\text { moribund patients. }\end{array}$ & $\begin{array}{l}\text { Pregnant or lactating } \\
\text { women, patients with } \\
\text { selenium intoxication, } \\
\text { individuals with } \\
\text { infections for which } \\
\text { guidelines recommend } \\
\text { a longer duration of } \\
\text { antimicrobial therapy, } \\
\text { immunocompromised } \\
\text { patients, and those } \\
\text { without commitment for } \\
\text { full therapy or where } \\
\text { death was imminent } \\
\text { owing to coexisting } \\
\text { diseases }\end{array}$ \\
\hline $\begin{array}{l}\text { ABx were discontinued } \\
\text { when PCT }<90 \% \text { of the } \\
\text { peak value or an absolute } \\
\text { value }<0.25 \mu \mathrm{g} / \mathrm{L}\end{array}$ & $\begin{array}{l}\text { ABx were discontinued } \\
\text { when } \mathrm{PCT}<90 \% \text { of the } \\
\text { peak value or an absolute } \\
\text { value }<0.5 \mu \mathrm{g} / \mathrm{L}\end{array}$ & $\begin{array}{l}\text { ABx were not to } \\
\text { be started or was } \\
\text { to be halted when } \\
\text { PCT }<0.25 \mu \mathrm{g} / \mathrm{L} \text {, strongly } \\
\text { discouraged when PCT } \\
\text { was } \geq 0.25 \text { to }<0.5 \mu \mathrm{g} / \mathrm{L} \text {, } \\
\text { recommended when } \\
\text { PCT } \geq 0.5 \text { to }<5 \mu \mathrm{g} / \mathrm{L} \text {, } \\
\text { strongly recommended } \\
\text { when } \mathrm{PCT} \geq 5 \mu \mathrm{g} / \mathrm{L}\end{array}$ & $\begin{array}{l}\text { ABx were discontinued } \\
\text { when } \mathrm{PCT} \text { value } \leq 0.5 \\
\mu \mathrm{g} / \mathrm{L}, \\
\mathrm{ABx} \text { were continued } \\
\text { when } \mathrm{PCT} \text { value } \geq 2 \mu \mathrm{g} / \mathrm{L}\end{array}$ & $\begin{array}{l}\text { ABx were discontinued } \\
\text { when PCT level }<0.10 \\
\mu \mathrm{g} / \mathrm{L} \text { or }<90 \% \text { of the peak } \\
\text { concentration }\end{array}$ & $\begin{array}{l}\text { ABx were discontinued } \\
\text { when PCT value }<80 \% \\
\text { of the peak value or an } \\
\text { absolute value } \leq 0.5 \mu \mathrm{g} / \mathrm{L}\end{array}$ & $\begin{array}{l}\text { ABx were discontinued } \\
\text { when PCT value }<50 \% \\
\text { of the peak value or an } \\
\text { absolute value } \leq 1.0 \mu \mathrm{g} / \mathrm{L}\end{array}$ \\
\hline $\begin{array}{l}\text { ABx were based on } \\
\text { the national guidelines } \\
\text { and the discretion of } \\
\text { attending physicians. }\end{array}$ & $\begin{array}{l}\text { ABx were based on the } \\
\text { physician following } \\
\text { the PCTprotocol after a } \\
\text { lecture about PCT and } \\
\text { the evidence about it. }\end{array}$ & $\begin{array}{l}\text { ABx were based on } \\
\text { the physician' own } \\
\text { assessment }\end{array}$ & $\begin{array}{l}\text { ABx were based on the } \\
\text { physician' antibiotic } \\
\text { empiric therapy }\end{array}$ & $\begin{array}{l}\text { ABx were based on the } \\
\text { local guidelines a treating } \\
\text { clinician were allowed } \\
\text { to overrule the algorithm } \\
\text { as clinically } \\
\text { indicated. }\end{array}$ & $\begin{array}{l}\text { ABx were based on } \\
\text { the local or national } \\
\text { guidelines and according } \\
\text { to the discretion of } \\
\text { attending physicians. }\end{array}$ & $\begin{array}{l}\text { ABx were based on the } \\
\text { local Guidelines }\end{array}$ \\
\hline $42 / 40$ & $42 / 39$ & $30 / 28$ & $30 / 30$ & $196 / 198$ & $761 / 785$ & $552 / 537$ \\
\hline
\end{tabular}

ICU, intensive care unit; PCT, procalcitonin; P, prospective; PG, parallel group; OL, open label; SB, single-blind; MC, multi-center; SC, single-center; ABx, antibiotics. 
Table 2. Outcome data of the studies included in the Meta-analysis: Procalcitonin-guided algorithms vs. Routine practice

\begin{tabular}{|c|c|c|c|c|c|c|c|c|c|c|c|}
\hline Study (reference) & $\begin{array}{l}\text { otal duration of } \\
\text { ntibiotic treatment, } \\
\text { lays, Mean (SD) }\end{array}$ & \multicolumn{2}{|c|}{$\begin{array}{l}\text { Length of ICU stay, } \\
\text { days, Mean (SD) }\end{array}$} & \multicolumn{2}{|c|}{$\begin{array}{l}\text { Length of hospital } \\
\text { stay, days, Mean (SD) }\end{array}$} & \multicolumn{2}{|c|}{ ICU mortality, n (\%) } & \multicolumn{2}{|c|}{$\begin{array}{l}\text { Hospital mortality, } \\
\text { n (\%) }\end{array}$} & $\begin{array}{l}\text { 28-day mortality, } \\
\text { n (\%) }\end{array}$ & $\begin{array}{l}\text { Rate of clinical cure, } \\
\text { n (\%) }\end{array}$ \\
\hline $\begin{array}{l}\text { Svoboda } \\
{[22]}\end{array}$ & NA & \multicolumn{2}{|c|}{$16.1 \pm 6.9$ vs. $19.4 \pm 8.9$} & \multicolumn{2}{|l|}{ NA } & \multicolumn{2}{|l|}{ NA } & \multicolumn{2}{|l|}{ NA } & $10(26 \%)$ vs. $13(38 \%)$ & NA \\
\hline $\begin{array}{l}\text { Nobre } \\
{[19]}\end{array}$ & NA & \multicolumn{2}{|c|}{$4(1-21)$ vs. $7(1-91)^{\mathrm{a}}$} & \multicolumn{2}{|c|}{$\begin{array}{l}17(3-96) \text { vs. } 23.5(5- \\
44)^{\mathrm{a}}\end{array}$} & \multicolumn{2}{|l|}{ NA } & \multicolumn{2}{|l|}{$\begin{array}{l}9(23.1 \%) \text { vs. } \\
9(22.5 \%)\end{array}$} & $8(20.5 \%)$ vs. $8(20 \%)$ & $\begin{array}{l}31(79.4 \%) \text { vs. } \\
32(80 \%)\end{array}$ \\
\hline \begin{tabular}{|l} 
Schroeder \\
{$[20]$}
\end{tabular} & $6.6 \pm 1.1$ vs. $8.3 \pm 0.7$ & \multicolumn{2}{|c|}{$16.4 \pm 8.3$ vs. $16.7 \pm 5.6$} & \multicolumn{2}{|l|}{ NA } & \multicolumn{2}{|l|}{ NA } & \multicolumn{2}{|l|}{$\begin{array}{l}3(21.4 \%) \text { vs. } \\
3(23.1 \%)\end{array}$} & NA & NA \\
\hline $\begin{array}{l}\text { Hochreiter } \\
{[14]}\end{array}$ & $5.9 \pm 1.7$ vs. $7.9 \pm 0.5$ & \multicolumn{2}{|c|}{$\begin{array}{l}15.5 \pm 12.5 \mathrm{vs} . \\
17.7 \pm 10.1\end{array}$} & \multicolumn{2}{|l|}{ NA } & \multicolumn{2}{|l|}{ NA } & \multicolumn{2}{|l|}{$\begin{array}{l}\text { 15(26.3\%)vs. } \\
14(26.4 \%)\end{array}$} & NA & NA \\
\hline $\begin{array}{l}\text { Bouadma } \\
{[12]}\end{array}$ & $10.3 \pm 7.7$ vs. $13.3 \pm 7.6$ & \multicolumn{2}{|c|}{$\begin{array}{l}15.9 \pm 16.1 \text { vs. } \\
14.4 \pm 14.1\end{array}$} & \multicolumn{2}{|c|}{$\begin{array}{l}26.1 \pm 19.3 \text { vs. } \\
26.4 \pm 18.3\end{array}$} & \multicolumn{2}{|l|}{ NA } & NA & & $\begin{array}{l}65(21.2 \%) \text { vs. } \\
64(20.4 \%)\end{array}$ & NA \\
\hline $\begin{array}{l}\text { Layios } \\
{[16]}\end{array}$ & JA & $7(4-16$ & 6) vs. $7(4-18)^{a}$ & NA & & $\begin{array}{l}56(21.7 \%) v s \\
53(21.1 \%)\end{array}$ & & NA & & & NA \\
\hline \begin{tabular}{|l} 
Liu BH \\
{$[17]$}
\end{tabular} & $.1 \pm 0.3$ vs. $9.3 \pm 0.3$ & $12.0 \pm 2$ & 2.9 vs. $14.0 \pm 2.7$ & $27.0 \pm 4.9$ & vs. $32.0 \pm 5.4$ & NA & & NA & & $\begin{array}{l}6(14.3 \%) \text { vs. } \\
5(12.5 \%)\end{array}$ & $\begin{array}{l}33(78.5 \%) v s . \\
34(85 \%)\end{array}$ \\
\hline \begin{tabular}{|l} 
Deliberato \\
{$[13]$}
\end{tabular} & $0(3-39)$ vs. $11(2-45)^{\mathrm{a}}$ & $3.5(1-$ & $-57)$ vs. $3(1-28)^{a}$ & $\begin{array}{l}11(3-547 \\
228)^{\mathrm{a}}\end{array}$ & vs. 11(2- & $1(2.4 \%)$ vs. & $4(10.3 \%)$ & $2(4.8 \%)$ vs. $4(10$ & $.3 \%)$ & NA & NA \\
\hline \begin{tabular}{|l} 
Annane \\
{$[10]$}
\end{tabular} & $(2-5)$ vs. $5(3-5)^{a}$ & $\begin{array}{l}22(8-4 \\
60)^{\mathrm{a}}\end{array}$ & 42) vs. 23(10- & $\begin{array}{l}27(9-49) \\
69)^{\mathrm{a}}\end{array}$ & vs. 33(11- & $7(23 \%)$ vs. 1 & 10(33\%) & $7(23 \%)$ vs. $10(3$ & $3 \%)$ & NA & NA \\
\hline $\begin{array}{l}\text { Najafi } \\
{[18]}\end{array}$ & 28 vs. 320 & $4(2-20$ & $0)$ vs. $6(2-28)^{a}$ & $20(8-44)$ & vs. $22(6-65)^{a}$ & NA & & $5(16.6 \%)$ vs. $4(1$ & $3.3 \%)$ & NA & $\begin{array}{l}27(90 \%) \mathrm{vs} . \\
26(86.6 \%)\end{array}$ \\
\hline $\begin{array}{l}\text { Shehabi } \\
{[21]}\end{array}$ & $(6-20)$ vs. $11(6-22)^{a}$ & $6(3-9$. & $.5)$ vs. $6(4-10)^{\mathrm{a}}$ & $\begin{array}{l}15(9-29) \\
32)^{\mathrm{a}}\end{array}$ & vs. $17(10-$ & $21(11 \%) v s$. & $15(8 \%)$ & $\begin{array}{l}30(16 \%) \text { vs. } 26 \\
(13 \%)\end{array}$ & & NA & NA \\
\hline $\begin{array}{r}\text { Jong } \\
{[15]} \\
\end{array}$ & $(3-9)$ vs. $7(4-11)^{a}$ & $8.5(5-$ & 17) vs. $9(4-17)^{\mathrm{a}}$ & $\begin{array}{l}22(13-39 \\
22(12-40\end{array}$ & 3) vs. & NA & & NA & & $\begin{array}{l}\text { 149(19.6\%) vs. } \\
196(25.0 \%)\end{array}$ & NA \\
\hline $\begin{array}{l}\text { Bloos } \\
{[11]}\end{array}$ & NA & $12(6-2$ & 24)vs. 11(6-21) & $\begin{array}{l}29(18-46 \\
44)^{\mathrm{a}}\end{array}$ & vs. 26(16- & NA & & NA & & $\begin{array}{l}140(25.6 \%) \mathrm{vs} . \\
149(28.2 \%)\end{array}$ & NA \\
\hline Study (reference) & Annane [10] & & Bloos [11] & & Bouadma [12 & & Deliberat & o [13] & Hochr & reiter [14] & ong [15] \\
\hline $\begin{array}{l}\text { Bias component } \\
\text { Sequence generation and } \\
\text { allocation concealment }\end{array}$ & $\begin{array}{l}\text { Randomised in a 1: } \\
\text { ratio according to a } \\
\text { computer-generatec } \\
\text { list. Randomisation } \\
\text { was centralised thr } \\
\text { a secured website } \\
\text { and performed by a } \\
\text { independent statisti } \\
\text { and was stratified } \\
\text { the centre and acco } \\
\text { to whether patients } \\
\text { underwent surgery } \\
\text { past } 48 \mathrm{~h} \text {. }\end{array}$ & $\begin{array}{l}1 \\
\text { d } \\
\text { ough } \\
\text { ician, } \\
\text { by } \\
\text { rding } \\
\text { in the }\end{array}$ & $\begin{array}{l}\text { An allocation ra } \\
1: 1 \text { by use of a c } \\
\text { randomization } \mathrm{x} \\
\text { server, Randomi } \\
\text { was stratified by } \\
\text { centre, sex, and } \\
\text { severity. }\end{array}$ & $\begin{array}{l}\text { tio of } \\
\text { central } \\
\text { veb } \\
\text { ization } \\
\text { ystudy } \\
\text { sepsis }\end{array}$ & $\begin{array}{l}\text { Independent, } \\
\text { computer-ge } \\
\text { randomisatio } \\
\text { was used to } 1 \\
\text { assign patien } \\
\text { ratio } \square \text { Patien } \\
\text { stratified by } \\
\text { random bloc } \\
4 \text {, or } 6 \text {; }\end{array}$ & $\begin{array}{l}\text { centralised, } \\
\text { enerated } \\
\text { on sequence } \\
\text { randomly } \\
\text { ats in a } 1: 1 \\
\text { ts were } \\
\text { centre with } \\
\text { k sizes of } 2 \text {, }\end{array}$ & $\begin{array}{l}2 \text { of the a } \\
\text { would ran } \\
\text { folder fro } \\
\text { containin } \\
(50 \text { "PCT } \\
\text { "control g }\end{array}$ & $\begin{array}{l}\text { uthors } \\
\text { ndomly draw } 1 \\
\text { m a black box } \\
\text { g } 100 \text { folders } \\
\text { group" and } 50 \\
\text { group"). }\end{array}$ & $\begin{array}{l}\text { Patient } \\
\text { assign } \\
\text { guided } \\
\text { withou } \\
\text { details }\end{array}$ & $\begin{array}{l}\text { ts were randomly } \\
\text { hed to either a PCT- } \\
\mathrm{d} \text { or control group } \\
\text { ut further reported } \\
\text { s }\end{array}$ & $\begin{array}{l}\text { andomised in a } 1: 1 \\
\text { atio according to a } \\
\text { omputer-generated } \\
\text { st. Randomisation was } \\
\text { ratified according to } \\
\text { eatment centre }\end{array}$ \\
\hline Blinding & $\begin{array}{l}\text { Using permutation } \\
\text { blocks, the size of } \\
\text { remained unknown } \\
\text { to the investigators } \\
\text { In the control arm, } \\
\text { patients, physicians } \\
\text { nurses, investigator } \\
\text { study coordinators, } \\
\text { the statistician and } \\
\text { sponsor remained b } \\
\text { to PCT levels throu } \\
\text { the study. }\end{array}$ & $\begin{array}{l}\text { which } \\
\text { the } \\
\text { linded } \\
\text { ighout }\end{array}$ & No & & $\begin{array}{l}\text { Investigators } \\
\text { masked } \\
\text { to assignmen } \\
\text { not after, ran } \\
\text { treatment ass } \\
\text { were not mas } \\
\text { investigators } \\
\text { unaware of a } \\
\text { outcomes du } \\
\text { study. }\end{array}$ & $\begin{array}{l}\text { were } \\
\text { nt before, but } \\
\text { adomisation, } \\
\text { signments } \\
\text { sked, all } \\
\text { were } \\
\text { aggregate } \\
\text { iring the }\end{array}$ & No & & No & & To \\
\hline Incomplete outcome data & $\begin{array}{l}3 \text { patients withdrew } \\
\text { informed consent, } 1 \\
\text { from PCT-Group, } 2 \\
\text { Control-Group }\end{array}$ & from & $\begin{array}{l}\text { Almost } 1 \text { every } \\
\text { screened patient } \\
\text { excluded from th }\end{array}$ & $\begin{array}{l}5 \\
\text { ts were } \\
\text { he study }\end{array}$ & $\begin{array}{l}2 \text { patients wi } \\
\text { informed con } \\
\text { each group }\end{array}$ & $\begin{array}{l}\text { ithdrew } \\
\text { nsent, } 1 \text { from }\end{array}$ & $\begin{array}{l}\text { No patien } \\
\text { follow-up }\end{array}$ & at was lost to & $\begin{array}{l}\text { Almos } \\
\text { screen } \\
\text { exclud }\end{array}$ & $\begin{array}{l}\text { st } 1 \text { every } 4 \\
\text { hed patients were } \\
\text { ded from the study }\end{array}$ & $\begin{array}{l}9 \text { patients withdrew } \\
\text { om the study, } 15 \text { from } \\
\text { CT-group, } 14 \text { from } \\
\text { ontrol-group. }\end{array}$ \\
\hline $\begin{array}{l}\text { Selective outcome } \\
\text { reporting }\end{array}$ & Extensive reporting & & Extensive report & ting & Extensive re & porting & Extensive & reporting & Limite & ed reporting & xtensive reporting \\
\hline Other sources of bias & $\begin{array}{l}\text { In control-group, th } \\
\text { physicians were fre } \\
\text { decide to continue } \\
\text { change the antibiot } \\
\text { clinical judgment }\end{array}$ & $\begin{array}{l}\text { ore } \\
\text { or } \\
\text { ics on }\end{array}$ & $\begin{array}{l}\text { No data on adeq } \\
\text { antimicrobial the }\end{array}$ & $\begin{array}{l}\text { quacy of } \\
\text { lerapy }\end{array}$ & $\begin{array}{l}\text { In control gro } \\
\text { investigators } \\
\text { to decide the } \\
\text { duration of an } \\
\text { treatment bas } \\
\text { own assessm } \\
\text { infection's cli }\end{array}$ & $\begin{array}{l}\text { oup, } \\
\text { were free } \\
\text { optimum } \\
\text { ntibiotic } \\
\text { sed on their } \\
\text { ent of the } \\
\text { inical course, }\end{array}$ & $\begin{array}{l}\text { not all att } \\
\text { physician } \\
\text { participat }\end{array}$ & $\begin{array}{l}\text { tending } \\
\text { is agreed to } \\
\text { te. }\end{array}$ & $\begin{array}{l}\text { No dat } \\
\text { antimi }\end{array}$ & $\begin{array}{l}\text { ta on adequacy of } \\
\text { icrobial therapy }\end{array}$ & $\begin{array}{l}\text { is unclear if there was } \\
\text { rossover between the } \\
\text { no groups }\end{array}$ \\
\hline Quality score & High & & High & & High & & High & & Low & & ligh \\
\hline
\end{tabular}

ICU, intensive care unit; NA, not available/applicable; ${ }^{a}$ These values are referred to median (range) instead of mean(SD). 
Table 3. Assessment of the methodologic aspects of the included studies

\begin{tabular}{|c|c|c|c|c|c|c|}
\hline $\begin{array}{l}\text { Layios } \\
{[16]}\end{array}$ & $\begin{array}{l}\text { Liu BH } \\
{[17]}\end{array}$ & $\begin{array}{l}\text { Najafi } \\
{[18]}\end{array}$ & $\begin{array}{l}\text { Nobre } \\
{[19]}\end{array}$ & $\begin{array}{l}\text { Schroeder } \\
{[20]}\end{array}$ & $\begin{array}{l}\text { Shehabi } \\
{[21]}\end{array}$ & $\begin{array}{l}\text { Svoboda } \\
{[22]}\end{array}$ \\
\hline $\begin{array}{l}\text { Patients were randomly } \\
\text { assigned to either a } \\
\text { PCT-guided or control } \\
\text { group antibiotic regimen } \\
\text { without further reported } \\
\text { details }\end{array}$ & $\begin{array}{l}\text { Randomised in a } 1: 1 \text { ratio } \\
\text { according to a computer- } \\
\text { generated list. }\end{array}$ & $\begin{array}{l}\text { Randomly divided into } \\
\text { two groups by computer } \\
\text { based } \\
\text { random number } \\
\text { generation, }\end{array}$ & $\begin{array}{l}\text { The randomization } \\
\text { was performed using a } \\
\text { computer-based random } \\
\text { number generation. } \\
\text { Allocation was issued } \\
\text { using opaque, sealed, } \\
\text { numbered } \\
\text { envelopes. }\end{array}$ & $\begin{array}{l}\text { Patients were randomly } \\
\text { assigned to either a PCT- } \\
\text { group or a control group } \\
\text { without further reported } \\
\text { details }\end{array}$ & $\begin{array}{l}\text { variable block } \\
\text { randomized } \\
\text { 1:1 via a secured central } \\
\text { study website into either } \\
\text { a PCT group) or control } \\
\text { group. Randomization } \\
\text { was stratified according } \\
\text { to the presence of septic } \\
\text { shock }\end{array}$ & $\begin{array}{l}\text { The randomization was } \\
\text { performed by means of } \\
\text { a computer generated } \\
\text { random number table } \\
\text { to generate a random } \\
\text { treatment list, treatment } \\
\text { regimens were included } \\
\text { in opaque sealed } \\
\text { numbered envelopes and } \\
\text { envelope with the lowest } \\
\text { number was always used } \\
\text { for consecutive patients }\end{array}$ \\
\hline $\begin{array}{l}\text { Physician was blinded to } \\
\text { PCT result, CT results in } \\
\text { the control group were } \\
\text { eventually } \\
\text { unblinded for the } \\
\text { statistical analysis }\end{array}$ & No & $\begin{array}{l}\text { Single-blind without } \\
\text { further reported details }\end{array}$ & No & No & $\begin{array}{l}\text { In control group, } \\
\text { clinicians were blinded } \\
\text { to the PCT levels, and } \\
\text { results were faxed } \\
\text { directly to the Clinical } \\
\text { Informatics and Data } \\
\text { Management Unit. }\end{array}$ & No \\
\hline $\begin{array}{l}\text { No patient was lost to } \\
\text { follow-up }\end{array}$ & $\begin{array}{l}\text { No patient was lost to } \\
\text { follow-up }\end{array}$ & $\begin{array}{l}\text { No patient was lost to } \\
\text { follow-up }\end{array}$ & $\begin{array}{l}12 \text { patients withdrew } \\
\text { from the study, } 8 \text { from } \\
\text { PCT-group,3 from } \\
\text { control-group. }\end{array}$ & $\begin{array}{l}\text { Almost } 1 \text { every } 5 \\
\text { screened patients were } \\
\text { excluded from the study }\end{array}$ & $\begin{array}{l}6 \text { patients withdrew from } \\
\text { the study, } 4 \text { from PCT- } \\
\text { group, } 2 \text { from control- } \\
\text { group. }\end{array}$ & $\begin{array}{l}\text { Almost } 1 \text { every } 6 \\
\text { screened patients were } \\
\text { excluded from the study }\end{array}$ \\
\hline Limited reporting & Limited reporting & Limited reporting & Extensive reporting & Limited reporting & Extensive reporting & Limited reporting \\
\hline $\begin{array}{l}\text { No serial determinations } \\
\text { of PCT throughout the } \\
\text { antibiotic course were } \\
\text { programmed upfront in } \\
\text { the design of the study }\end{array}$ & $\begin{array}{l}\text { No data on adequacy of } \\
\text { antimicrobial therapy }\end{array}$ & $\begin{array}{l}\text { In control-group,the } \\
\text { physicians were free to } \\
\text { decide to continue or } \\
\text { change the antibiotics on } \\
\text { their own empiric }\end{array}$ & $\begin{array}{l}\text { "Algorithm overruling" } \\
\text { in the PCT group } \\
\text { occurred in } 6 \text { of } 31 \\
(19 \%) \text {, The study } \\
\text { investigators did not } \\
\text { interfere with the } \\
\text { duration of antibiotic } \\
\text { therapy in control-group }\end{array}$ & $\begin{array}{l}\text { Physician in charge was } \\
\text { always free to decide to } \\
\text { continue or change the } \\
\text { antibiotic regimen upon } \\
\text { clinical judgement. }\end{array}$ & $\begin{array}{l}\text { Treating clinicians could } \\
\text { overrule the algorithm as } \\
\text { clinically } \\
\text { indicated. }\end{array}$ & $\begin{array}{l}\text { It is unclear if there was } \\
\text { crossover between the } \\
\text { two groups }\end{array}$ \\
\hline Low & Low & High & High & Low & High & Low \\
\hline
\end{tabular}

of high quality $[10-13,15,18-19,21]$ and five of low quality $[14,16-$ $17,20,22]$.

\section{Evidence synthesis}

\section{The total duration of antibiotic therapy}

Eight RCTs $(10,12-15,17,20-21)$ in this Meta-analysis provided data on the duration of antibiotic therapy. Significant heterogeneity could be detected between these studies $\left(\mathrm{Tau}^{2}=0.47 ; \mathrm{Chi}^{2}=182.58\right.$, $\left.\mathrm{df}=7 ; P<0.00001 ; \mathrm{I}^{2}=96 \%\right)$. The combined estimate for the duration of antibiotic therapy based on the random-effects model showed statistically significant difference between the PCT and the routine practice group (2919 patients; REM; $\mathrm{MD}=-1.60 ; 95 \% \mathrm{CI},-2.14$ to -1.06 ; $\mathrm{Z}=5.79 ; P<0.00001)$ [Figure 2].

The subgroup analysis between the developed countries $(10,12$, $14-15,20-21)$ and the developing countries $(13,17)$ showed that the duration of antibiotic therapy had significant difference both in the developed countries(2756 patients; REM; $\mathrm{MD}=-1.72 ; 95 \% \mathrm{CI},-2.52$ to -0.93; $Z=4.27 ; P<0.00001$ ) and the developing countries(163patients; REM; $M D=-1.20 ; 95 \% C I,-1.33$ to $-1.07 ; Z=18.11 ; P<0.00001$ ).

Sensitivity analysis including only the highest quality studies (10, $12-13,15,21)$ led to similar findings (2700 patients; REM; $M D=-1.65$; 95\%CI, -2.78 to $-0.51 ; \mathrm{Z}=2.85 ; P=0.004)$.

\section{Length of intensive care unit stay}

Thirteen RCTs [10-22] in this Meta-analysis provided data on the length of ICU stay. Significant heterogeneity could be detected between these studies $\left(\mathrm{Tau}^{2}=0.37 ; \mathrm{Chi}^{2}=75.61, \mathrm{df}=12 ; P<0.00001 ; \mathrm{I}^{2}=84 \%\right)$. The combined estimate for the length of ICU stay based on the random- effects model showed no statistically significant difference between the PCT and the routine practice group (4728 patients; REM; $\mathrm{MD}=-0.23$; 95\%CI, -0.76 to $0.31 ; \mathrm{Z}=0.83 ; P=0.41$ ) [Figure 3 ].

The subgroup analysis between the developed countries(10-12, $14-16,19-21)$ and the developing countries $[13,17,18,22]$ showed that the length of ICU stay had significant difference in the developing countries(295 patients; REM; $\mathrm{MD}=-1.98 ; 95 \% \mathrm{CI},-3.02$ to -0.94 ; $\mathrm{Z}=3.73 \quad \mathrm{P}=0.0002)$, but no significant difference in the developed countries(4433patients; REM; $\mathrm{MD}=0.11 ; 95 \% \mathrm{CI},-0.44$ to $0.65 ; \mathrm{Z}=0.39$; $P=0.70)$.

Sensitivity analysis including only the highest quality studies (10$13,15,18-19,21)$ led to similar findings (3928 patients; REM; $M D=0.10$; $95 \% \mathrm{CI},-0.58$ to $0.78 ; \mathrm{Z}=0.29 ; P=0.77)$.

\section{Length of hospital stay}

Nine RCTs $(10-13,15,17-19,21)$ in this Meta-analysis provided data on the length of hospital stay. Significant heterogeneity could be detected between these studies $\left(\mathrm{Tau}^{2}=5.83 ; \mathrm{Chi}^{2}=162.36, \mathrm{df}=8\right.$; $\left.P<0.00001 ; \mathrm{I}^{2}=95 \%\right)$. The combined estimate for the length of hospital stay based on the random-effects model showed no statistically significant difference between the PCT and the routine practice group (4010 patients; REM; $\mathrm{MD}=-1.43 ; 95 \% \mathrm{CI},-3.45$ to $0.59 ; \mathrm{Z}=1.39 ; P=0.17$ ) [Figure 4].

The subgroup analysis between the developed countries(10-12, 15, $19,21)$ and the developing countries $[13,17,18]$ showed that the length of hospital stay had significant difference in the developing countries(223 patients; REM; $\mathrm{MD}=-4.65$; $95 \% \mathrm{CI},-6.75$ to -2.55 ; $\mathrm{Z}=4.33 ; P<0.00001$ ) ,but no significant difference in the developed countries(3787 patients; REM; $\mathrm{MD}=-0.66 ; 95 \% \mathrm{CI},-2.85$ to $1.54 ; \mathrm{Z}=0.59 ; P=0.56$ ). 


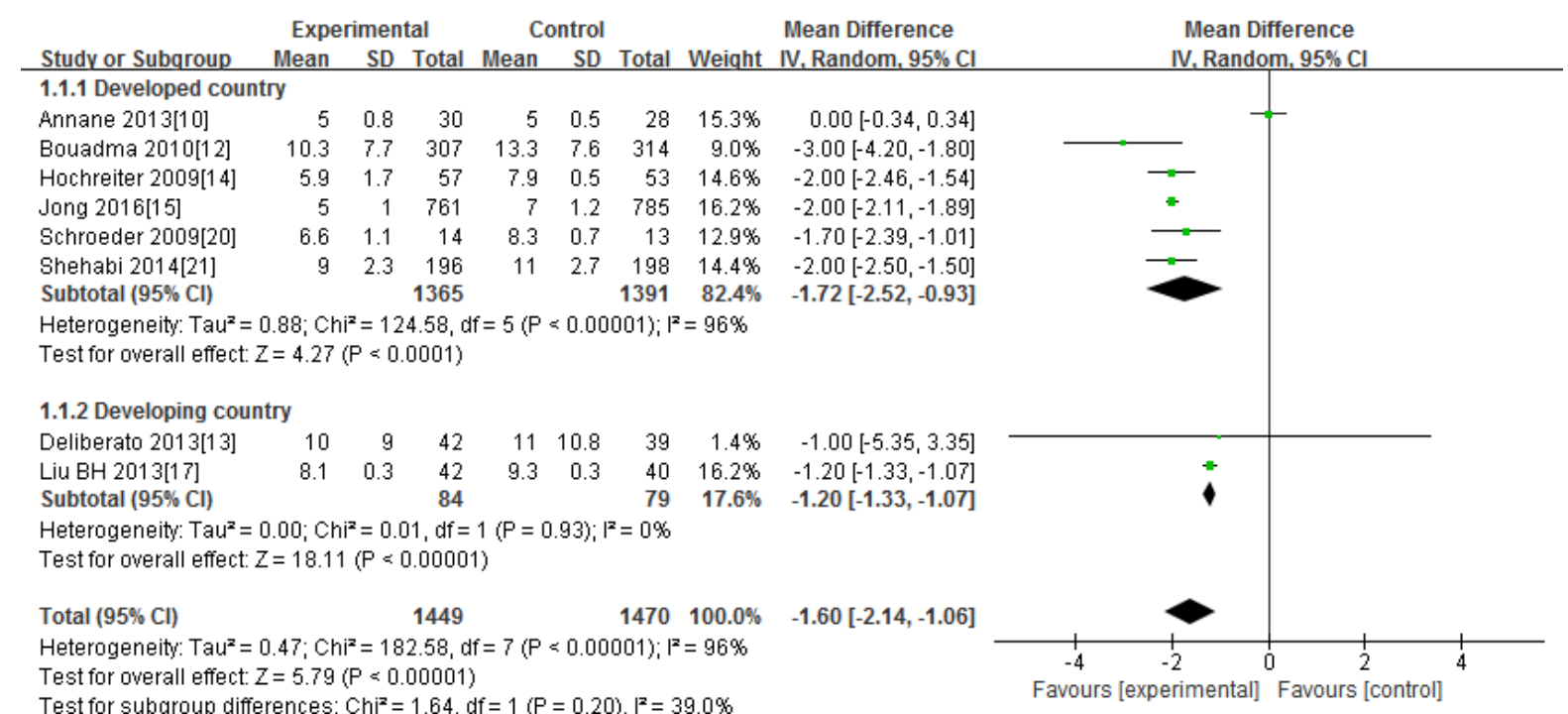

Figure 2. The duration of antibiotic therapy, days. forest plot showing the comparison of PCT-guided algorithms vs. routine practice. The size of each square represents the proportion of information provided by each study. The vertical line depicts the point of "no difference" between the two groups, and the horizontal lines correspond to the $95 \%$ confidence intervals (CIs). Diamonds represent the mean difference (MD) for all studies.

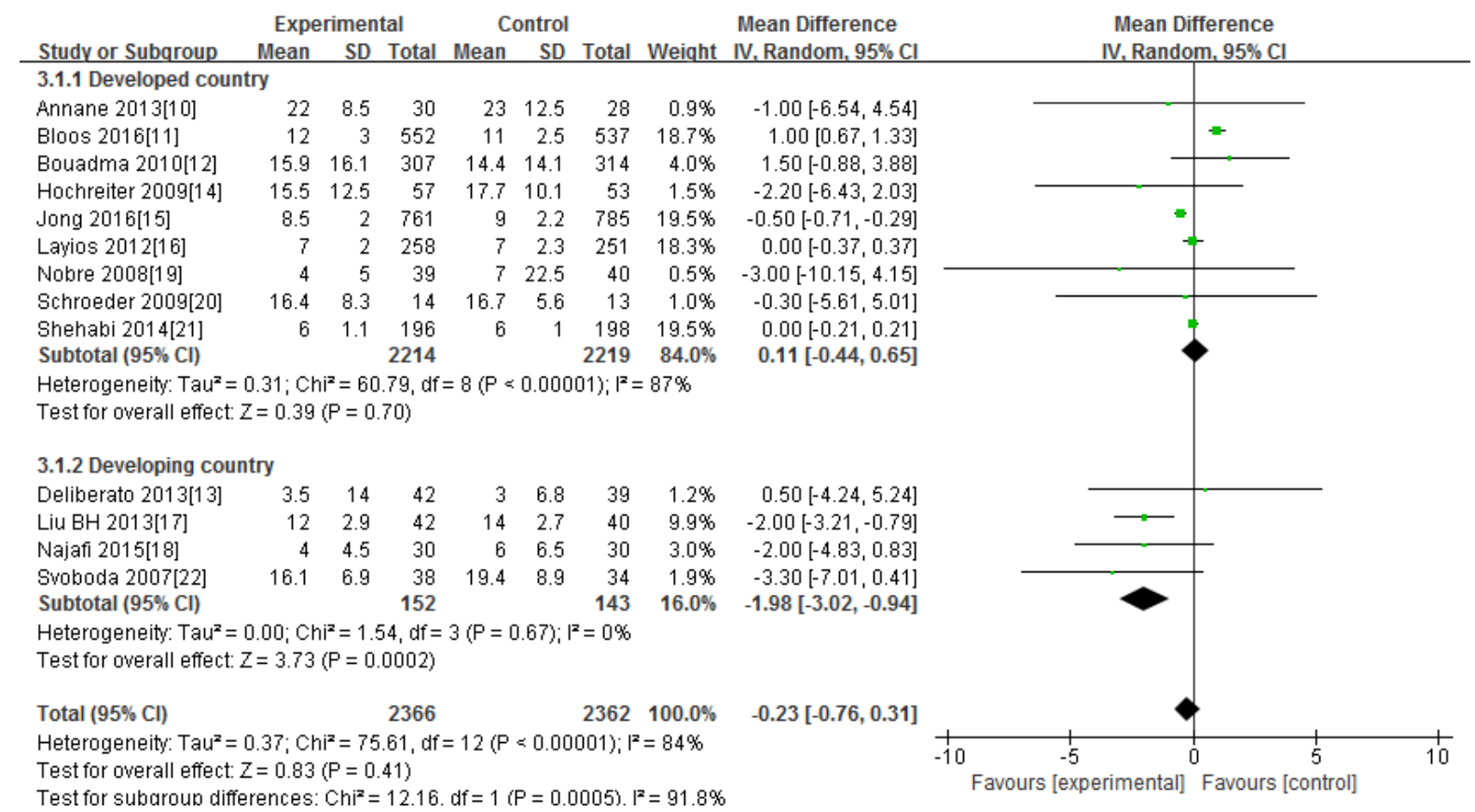

Figure 3. The length of ICU stay. forest plot showing the comparison of PCT-guided algorithms vs. routine practice. The size of each square represents the proportion of information provided by each study. The vertical line depicts the point of "no difference" between the two groups, and the horizontal lines correspond to the $95 \%$ confidence intervals (CIs). Diamonds represent the mean difference (MD) for all studies.

Sensitivity analysis including only the highest quality studies [10$19,21]$ led to similar findings (3928 patients; REM; $\mathrm{MD}=-0.75 ; 95 \% \mathrm{CI}$, -2.84 to $1.35 ; \mathrm{Z}=0.70 ; P=0.49)$.

\section{8-day mortality}

Six RCTs $[11,12,15,17,19,22]$ in this Meta-analysis provided data on the 28-day mortality. No significant heterogeneity could be detected between these studies $\left(\mathrm{Chi}^{2}=3.92, \mathrm{df}=5 ; P=0.56 ; \mathrm{I}^{2}=0 \%\right)$. The combined estimate for the 28-day mortality based on the fixed-effects model showed statistically significant difference between the PCT and the routine practice group (3476 patients; $\mathrm{FEM} ; \mathrm{MD}=0.86$; $95 \% \mathrm{CI}, 0.76$ to 0.97; $\mathrm{Z}=2.39 ; P=0.02$ ) [Figure 5].
The subgroup analysis between the developed countries $[11,12,15,19]$ and the developing countries $[17,22]$ showed that the 28-day mortality had significant difference in the developed countries (3322 patients; FEM; $\mathrm{MD}=0.87 ; 95 \% \mathrm{CI}, 0.76$ to 0.98 ; $\mathrm{Z}=2.29 ; P=0.02)$, but no significant difference in the developing countries $(154$ patients; $\mathrm{FEM} ; \mathrm{MD}=0.81 ; 95 \% \mathrm{CI}, 0.45$ to 1.45 ; $\mathrm{Z}=0.70 ; P=0.48)$.

Sensitivity analysis including only the highest quality studies $[11,12,15,19]$ led to similar findings (3322 patients; FEM; $M D=0.87$; 95\%CI, 0.76 to $0.98 ; \mathrm{Z}=2.29 ; P=0.02$ ). 


\begin{tabular}{|c|c|c|c|c|c|c|c|c|c|c|}
\hline \multirow[b]{2}{*}{ Study or Subqroup } & \multicolumn{3}{|c|}{ Experimental } & \multicolumn{3}{|c|}{ Control } & & \multirow{2}{*}{$\begin{array}{l}\text { Mean Difference } \\
\text { IV, Random, } 95 \% \mathrm{Cl}\end{array}$} & \multirow{2}{*}{\multicolumn{2}{|c|}{$\begin{array}{l}\text { Mean Difference } \\
\text { IV. Random, } 95 \% \mathrm{Cl}\end{array}$}} \\
\hline & Mean & SD & Total & Mean & SD & Total & Weight & & & \\
\hline \multicolumn{10}{|c|}{ 5.1.1 Developed country } & \multirow{8}{*}{ - } \\
\hline Annane $2013[10]$ & 27 & 10 & 30 & 33 & 14.5 & 28 & $6.4 \%$ & $-6.00[-12.45,0.45]$ & & \\
\hline Bloos 2016[11] & 29 & 4.7 & 552 & 26 & 4.7 & 537 & $18.0 \%$ & $3.00[2.44,3.56]$ & & \\
\hline Bouadma 2010[12] & 26.1 & 19.3 & 307 & 26.4 & 18.3 & 314 & $13.1 \%$ & $-0.30[-3.26,2.66]$ & & \\
\hline Jong 2016[15] & 22 & 4.4 & 761 & 22 & 4.7 & 785 & $18.1 \%$ & $0.00[-0.45,0.45]$ & & \\
\hline Nobre 2008[19] & 17 & 23.3 & 39 & 23.5 & 9.8 & 40 & $4.8 \%$ & $-6.50[-14.42,1.42]$ & & \\
\hline Shehabi 2014 [21] & 15 & 3.3 & 196 & 17 & 3.7 & 198 & $17.9 \%$ & $-2.00[-2.69,-1.31]$ &. & \\
\hline Subtotal $(95 \% \mathrm{Cl})$ & & & 1885 & & & 1902 & $78.2 \%$ & $-0.66[-2.85,1.54]$ & & \\
\hline \multicolumn{10}{|c|}{$\begin{array}{l}\text { Heterogeneity: } \operatorname{Tau}^{2}=5.29 ; \mathrm{Ch}^{2}=138.98, \mathrm{df}=5(P<0.00001) ; \mathrm{I}^{2}=96 \% \\
\text { Test for overall effect: } Z=0.59(P=0.56)\end{array}$} & \\
\hline \multirow{2}{*}{\multicolumn{11}{|c|}{ 5.1.2 Developing country }} \\
\hline Deliberato $2013[13]$ & 11 & & & & & & & & & \\
\hline Liu BH $2013[17]$ & 27 & 4.9 & 42 & 32 & 5.4 & 40 & $14.9 \%$ & $-5.00[-7.24,-2.76]$ & $=$ & \\
\hline Najafi 2015[18] & 20 & 9 & 30 & 22 & 15 & 30 & $6.6 \%$ & $-2.00[-8.26,4.26]$ & & \\
\hline Subtotal $(95 \% \mathrm{Cl})$ & & & 114 & & & 109 & $21.8 \%$ & $-4.65[-6.75,-2.55]$ & $\bullet$ & \\
\hline \multicolumn{11}{|c|}{$\begin{array}{l}\text { Heterogeneity: } \mathrm{Tau}^{2}=0.00 ; \mathrm{Chi}^{2}=0.82, \mathrm{df}=2(\mathrm{P}=0.66) ; \mathrm{I}^{2}=0 \% \\
\text { Test for overall effect: } Z=4.33(\mathrm{P}<0.0001)\end{array}$} \\
\hline Total $(95 \% \mathrm{Cl})$ & & & 1999 & & & 2011 & $100.0 \%$ & $-1.43[-3.45,0.59]$ & 4 & 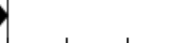 \\
\hline \multicolumn{9}{|c|}{ 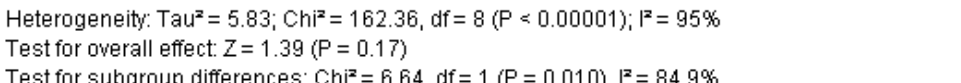 } & $\begin{array}{cc}-20 & -10 \\
\text { Favours [experimental] }\end{array}$ & $\begin{array}{ccc}0 & 1 & 1 \\
0 & 10 & 20 \\
\text { Favours [contr }\end{array}$ \\
\hline
\end{tabular}

Figure 4. The length of hospital stay. forest plot showing the comparison of PCT-guided algorithms vs. routine practice. The size of each square represents the proportion of information provided by each study. The vertical line depicts the point of "no difference" between the two groups, and the horizontal lines correspond to the $95 \%$ confidence intervals (CIs). Diamonds represent the mean difference (MD) for all studies.

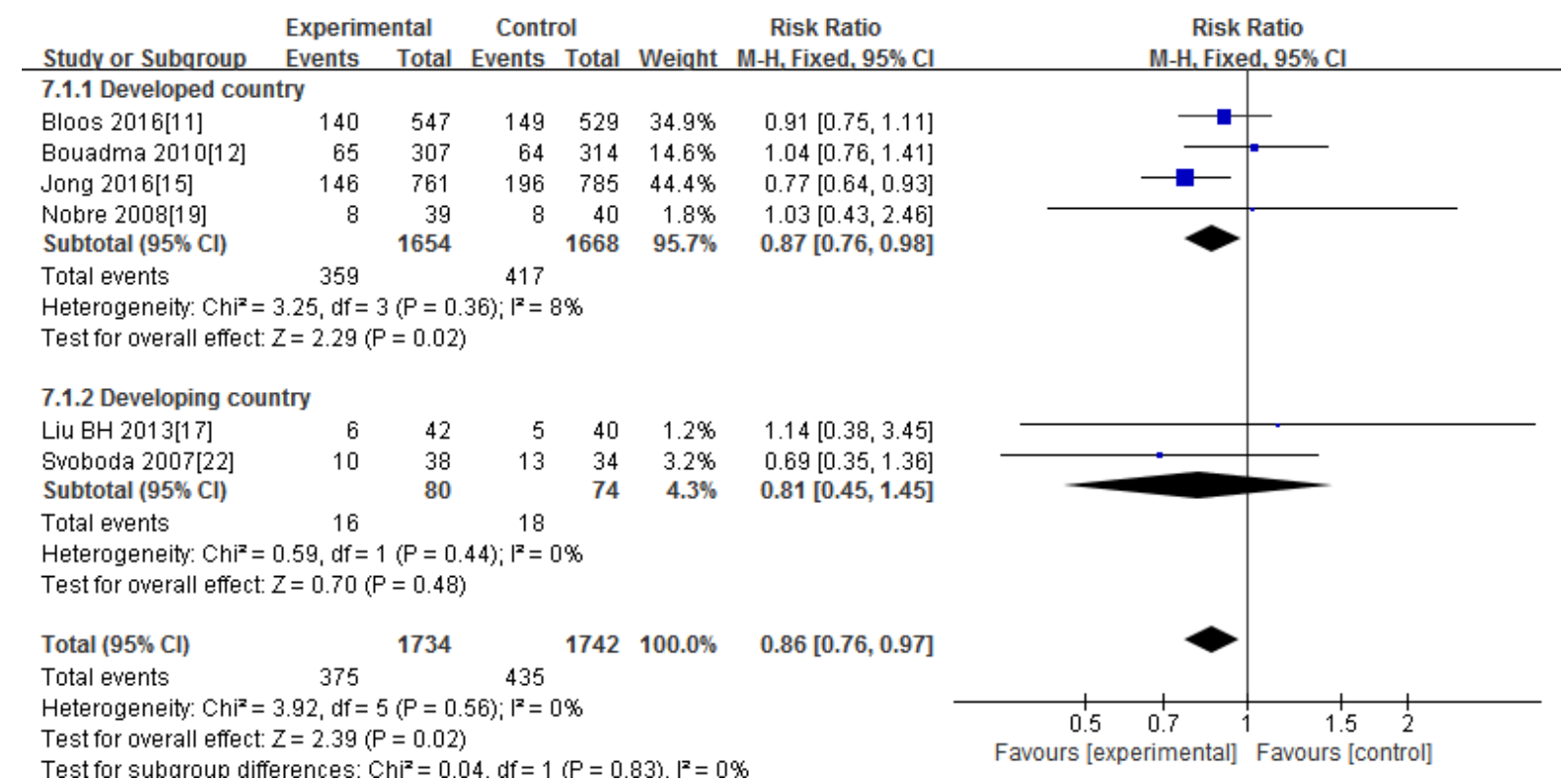

Figure 5. 28-day mortality. forest plot showing the comparison of PCT-guided algorithms vs. routine practice. The size of each square represents the proportion of information provided by each study. The vertical line depicts the point of "no difference" between the two groups, and the horizontal lines correspond to the $95 \%$ confidence intervals (CIs). Diamonds represent the mean difference (MD) for all studies.

\section{ICU mortality}

Four RCTs $[10,13,16,21]$ in this Meta-analysis provided data on the ICU mortality. No significant heterogeneity could be detected between these studies $\left(\mathrm{Chi}^{2}=3.80, \mathrm{df}=3 ; \quad P=0.28 ; \mathrm{I}^{2}=21 \%\right)$. The combined estimate for the ICU mortality based on the fixed-effects model showed no statistically significant difference between the PCT and the routine practice group (1045 patients; FEM; $\mathrm{MD}=1.01 ; 95 \% \mathrm{CI}, 0.77$ to 1.33 ; $\mathrm{Z}=0.10 ; P=0.92$ ) [Figure 6].

The subgroup analysis between the developed countries [10,16,21] and the developing countries [13] showed that the ICU mortality had no significant difference in the developed countries (964 patients; FEM;
$\mathrm{MD}=1.06 ; 95 \% \mathrm{CI}, 0.80$ to $1.39 ; \mathrm{Z}=0.39 ; P=0.70)$. There was only one study in the developing countries

Sensitivity analysis including only the highest quality studies $[10,13,21]$ led to similar findings (536 patients; $F E M ; M D=0.99 ; 95 \% \mathrm{CI}$, 0.62 to $1.59 ; \mathrm{Z}=0.04 ; P=0.97)$.

\section{In hospital mortality}

Seven RCTs $[10,13,14,18-21]$ in this Meta-analysis provided data on the in-hospital mortality. No significant heterogeneity could be detected between these studies $\left(\mathrm{Chi}^{2}=2.50, \mathrm{df}=6 ; P=0.87 ; \mathrm{I}^{2}=0 \%\right)$. The combined estimate for the in-hospital mortality based on the fixed- 


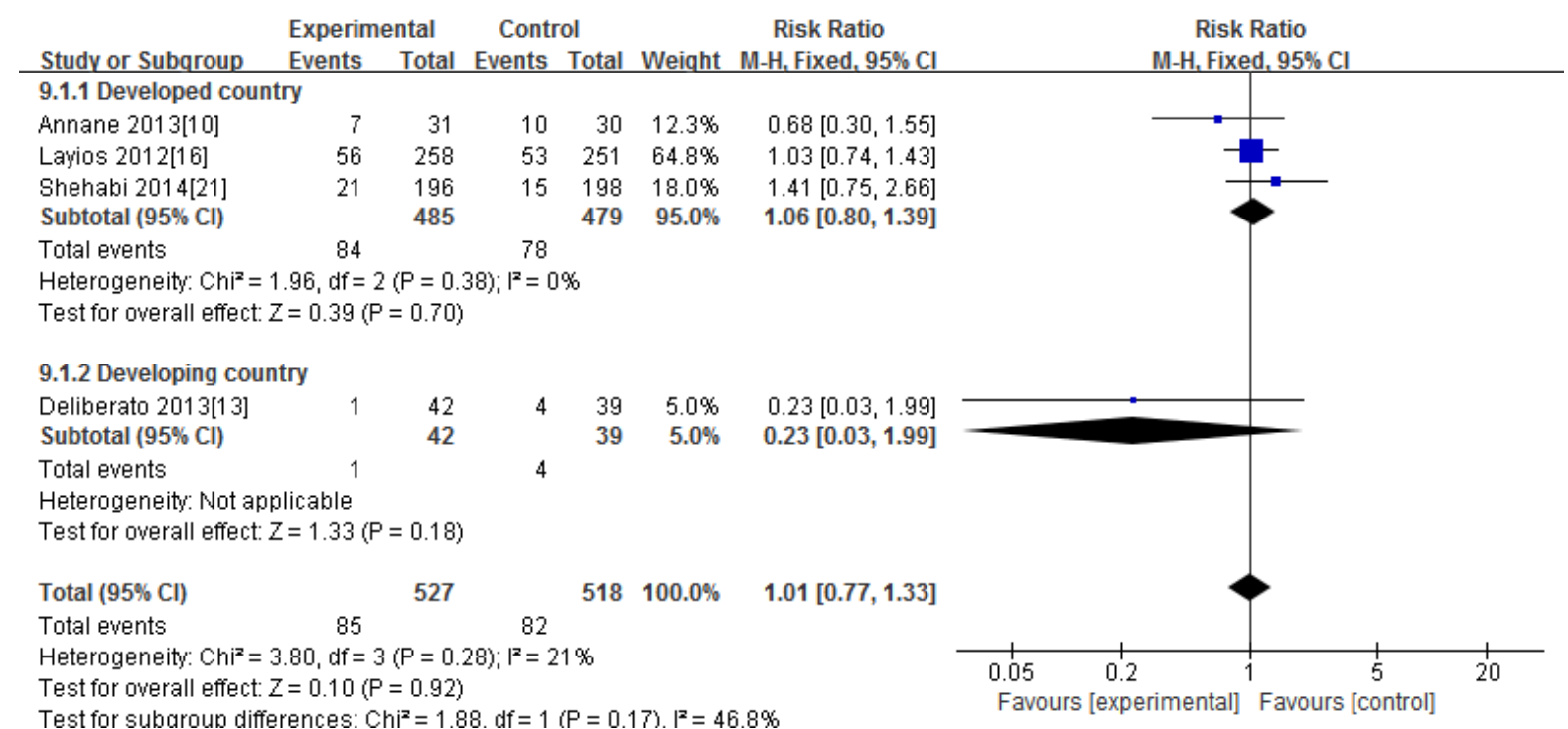

Figure 6. ICU mortality. forest plot showing the comparison of PCT-guided algorithms vs. routine practice. The size of each square represents the proportion of information provided by each study. The vertical line depicts the point of "no difference" between the two groups, and the horizontal lines correspond to the $95 \%$ confidence intervals (CIs). Diamonds represent the mean difference (MD) for all studies.

effects model showed no statistically significant difference between the PCT and the routine practice group (1096 patients; FEM; $\mathrm{MD}=1.05$; 95\%CI, 0.80 to $1.38 ; \mathrm{Z}=0.34 ; P=0.73$ ) [Figure 7].

The subgroup analysis between the developed countries [10,14,19$21]$ and the developing countries $[13,18]$ showed that the in hospital mortality had no significant difference both in the developed countries (955 patients; FEM; $\mathrm{MD}=1.07 ; 95 \% \mathrm{CI}, 0.80$ to $1.43 ; \mathrm{Z}=0.47 ; P=0.64$ ) and the developing countries ( 141 patients; FEM; $\mathrm{MD}=0.85$; $95 \% \mathrm{CI}$, 0.33 to $2.20 ; \mathrm{Z}=0.34 ; P=0.74$ ).

Sensitivity analysis including only the highest quality studies (10, $13,18-19,21)$ led to similar findings (675 patients; FEM; $M D=1.00$; 95\%CI, 0.74 to $1.41 ; \mathrm{Z}=0.00 ; P=1.00$ ).

\section{The rates of clinical cure}

Three RCTs (17-19) in this Meta-analysis provided data on the clinical cure. No significant heterogeneity could be detected between these studies $\left(\mathrm{Chi}^{2}=0.71, \mathrm{df}=2 ; \mathrm{P}=0.70 ; \mathrm{I}^{2}=0 \%\right.$ ). The combined estimate for the rates of clinical cure based on the fixed-effects model showed no statistically significant difference between the PCT and the routine practice group (221 patients; $\mathrm{FEM}$; $\mathrm{MD}=0.98 ; 95 \% \mathrm{CI}, 0.87$ to 1.10 ; $\mathrm{Z}=0.33 ; P=0.74$ ) [Figure 8].

The subgroup analysis between the developed countries [19] and the developing countries [17-18] showed that the rates of clinical cure had no significant difference in the developing countries (142 patients; FEM; $\mathrm{MD}=0.97 ; 95 \% \mathrm{CI}, 0.85$ to $1.12 ; \mathrm{Z}=0.38 ; P=0.70$ ). There was only one study in the developed countries.

Sensitivity analysis including only the highest quality studies [1819] led to similar findings ( 139 patients; FEM; $\mathrm{MD}=1.01 ; 95 \% \mathrm{CI}, 0.88$ to $1.17 ; \mathrm{Z}=0.18 ; P=0.85)$.

\section{Publication bias}

Thirteen studies [10-22] were included in this Meta-analysis. Assessment of publication bias using a funnel plot was presented in [Figure 9].

\section{Discussion}

Our Meta-analysis included thirteen randomized controlled studies involving 4728 ICU septic patients. By synthesizing data from these studies, we found that PCT-guided algorithm decreased the total duration of antibiotic therapy which is in accordance with the former studies [23-25]. Furthermore, we also found that PCT-guided algorithm reduced the 28-day mortality of septic patients in ICU, which is different from the former studies [26-27]. These results were also retained in the sensitivity analysis. As antibiotic consumption and acquired antimicrobial resistance had been shown to be associated with increased mortality and health care costs [28], clinicians may be encouraged to tailor the antibiotic therapy of septic patients in ICU according to the guidance of PCT, rather than the empiric rules.

However, our findings do not show a significant difference between the PCT-guided algorithm and the routine practice regarding the length of ICU or hospital stay, respectively. Besides, the ICU mortality, in hospital mortality and rates of clinical cure were not different between the PCU-guided group and the routine practice group, which is in accordance with the research of Christ Crain, et al. [29]. This finding provides some reassurance that PCT-guided therapy is safe and will not compromise the septic patients' clinical outcomes [30].

All studies in our Meta-analysis come from ten different countries. The developed countries include Switzerland, Germany, France, Belgium, Australian, Netherlands, the developing countries include Czech Republic, China, Brazil, Iran. The main finding of the subgroup showed that in the developed countries, The PCT-guided algorithm could decrease the total duration of antibiotic therapy and reduce the 28-day mortality of septic patients in ICU. While, in the developing countries, the PCT-guided algorithm could decrease the total duration of antibiotic therapy, the length of ICU and the length of hospital stay without reducing the 28-day mortality. Both in the developed countries and the developing countries, the PCT-guided algorithm may not improve the ICU mortality, the in hospital mortality and the rates of clinical cure.

This Meta-analysis has several limitations. First, not all included studies provided data on all outcomes. Second, the PCT-guided 


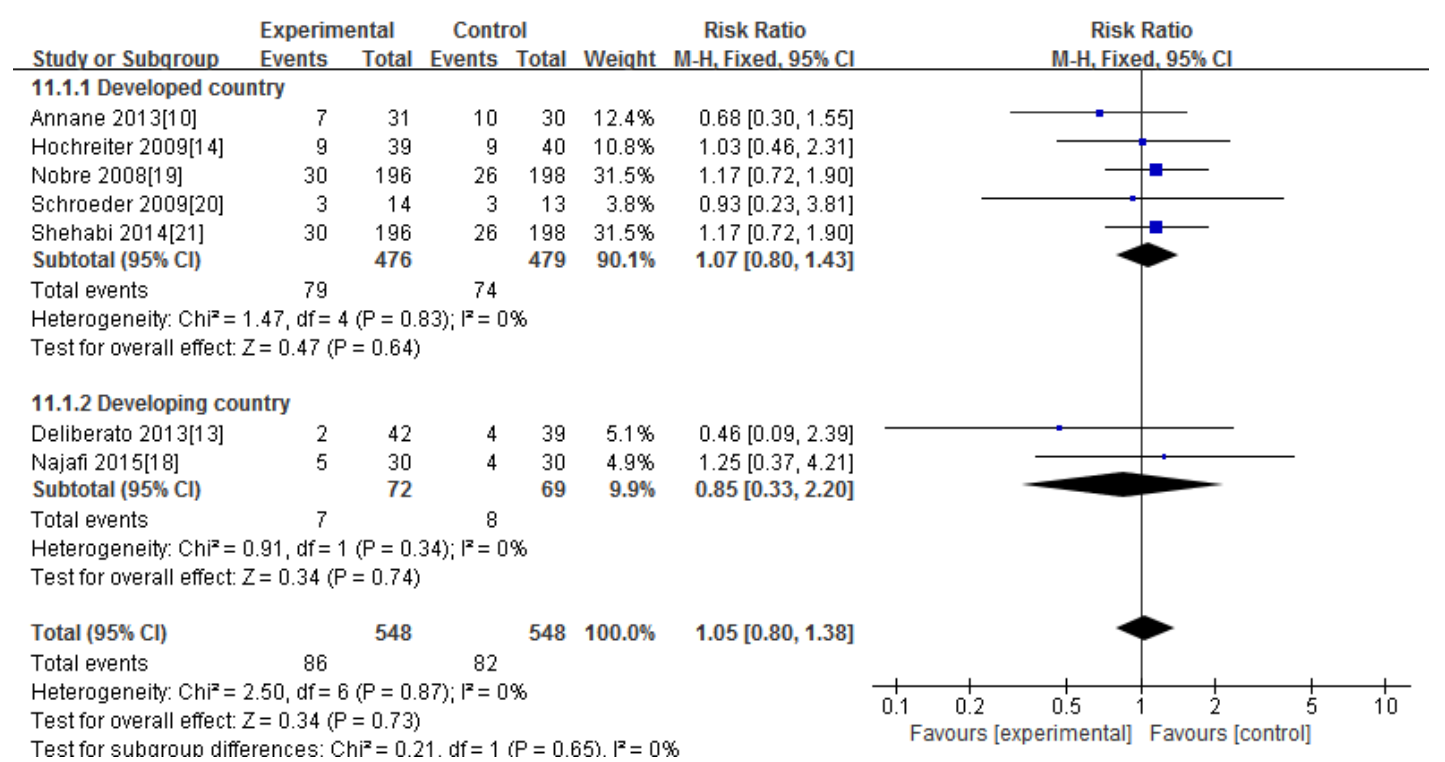

Figure 7. In hospital mortality. forest plot showing the comparison of PCT-guided algorithms vs. routine practice. The size of each square represents the proportion of information provided by each study. The vertical line depicts the point of "no difference" between the two groups, and the horizontal lines correspond to the $95 \%$ confidence intervals (CIs). Diamonds represent the mean difference (MD) for all studies.

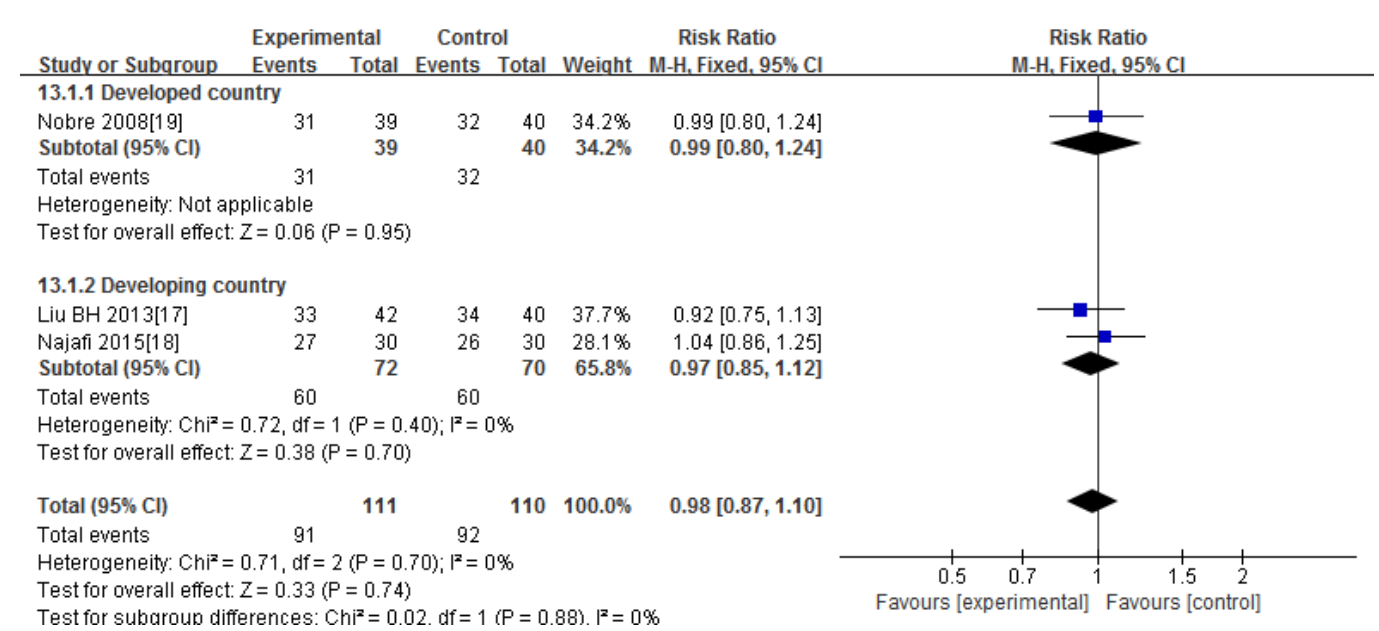

Figure 8. The rates of clinical cure. forest plot showing the comparison of PCT-guided algorithms vs. routine practice. The size of each square represents the proportion of information provided by each study. The vertical line depicts the point of "no difference" between the two groups, and the horizontal lines correspond to the $95 \%$ confidence intervals (CIs). Diamonds represent the mean difference (MD) for all studies.

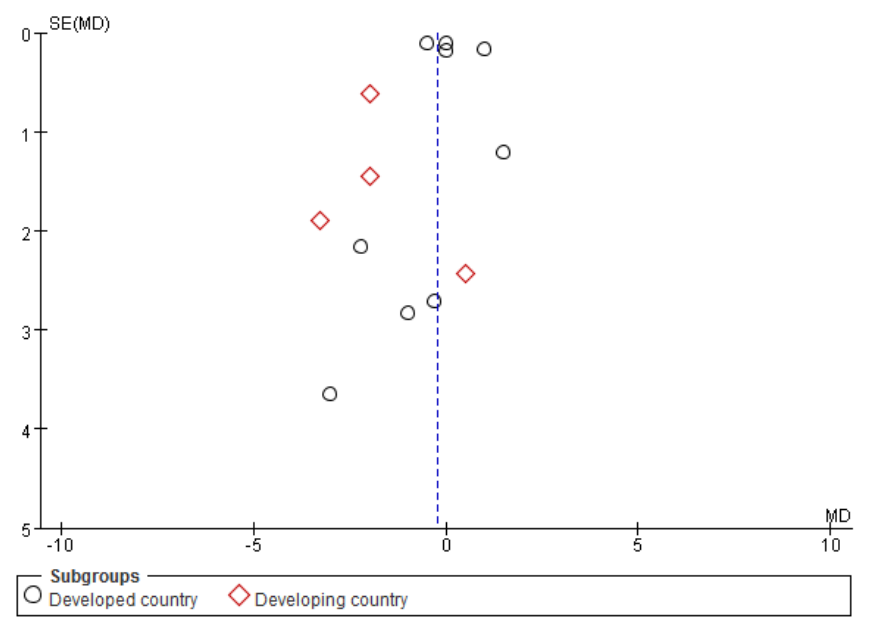

Figure 9. Funnel plot algorithms were very heterogeneous as far as the level of PCT chosen to make antibiotic therapy. Third, studies included in our Meta-analysis varied in study design and objectives.

In conclusion, the present available evidence seems to suggest that the implementation of PCT-guided algorithms for the antibiotic therapy of septic patients in ICU is associated with reduced the total antibiotic exposure and the 28-day mortality without the length of ICU and hospital stay, ICU mortality, in hospital mortality and rates of clinical cure. Further research is still necessary before the wide adoption of this strategy.

\section{References}

1. Levy MM, Fink MP, Marshall JC (2003) 2001 SCCM/ESICM/ACCP/ATS/SIS International Sepsis Definitions Conference. Crit Care Med 31: 1250-1256. [Crossref]

2. Dombrovskiy VY, Martin AA, Sunderram J (2007) Rapid increase in hospitalization and mortality rates for severe sepsis in the United States: A trend analysis from 1993 to 2003.Crit Care Med 35: 1244-1250. [Crossref] 
3. Martin GS, Mannino DM, Eaton S (2003) The epidemiology of sepsis in the United States from 1979 through 2000. N Engl J Med 348: 1546-1554. [Crossref]

4. Becker KL, Nyle'n ES, White JC (2004) Clinical review 167: Procalcitonin and the calcitonin gene family of peptides in inflammation, infection, and sepsis: A journey from calcitonin back to its precursors. J Clin Endocrinol Metab 89: 1512-1525. [Crossref]

5. Uzzan B, Cohen R, Nicolas P (2006) Procalcitonin as a diagnostic test for sepsis in critically ill adults and after surgery or trauma: A systematic review and meta-analysis. Crit Care Med 34: 1996-2003. [Crossref]

6. Anna Prkno, Christina Wacker, Frank M Brunkhorst (2013) Procalcitonin-guided therapy in intensive care unit patients with severe sepsis and septic shock - a systematic review and meta-analysis. Crit Care 17: 291

7. Petros Kopterides, Ilias I. Siempos, Iraklis Tsangaris (2010) Procalcitonin-guided algorithms of antibiotic therapy in the intensive care unit: A systematic review and meta-analysis of randomized controlled trials. Crit Care Med 38: 2229-2241. [Crossref]

8. Higgins JP, Green S. Cochrane Handbook for Systematic Reviews of Interventions (Version 5.1.0).

9. Higgins JP, Thompson SG, Deeks JJ (2003) Measuring inconsistency in meta-analyses. BMJ 327: 557-560. [Crossref]

10. Djillali Annane, Virginie Maxime, Jean Pierre Faller (2013) Procalcitonin levels to guide antibiotic therapy in adults with non-microbiologically proven apparent severe sepsis: a randomized controlled trial. BMJ Open 3: e002186. [Crossref]

11. F Bloos, E Trips, A Nierhaus (2016) Effect of Sodium Selenite Administration and Procalcitonin-Guided Therapy on Mortality in Patients With Severe Sepsis or Septic Shock: A Randomized Clinical Trial. JAMA Intern Med 176: 1266-1276.

12. L Bouadma, CE Luyt, F Tubach (2010) Use of procalcitonin to reduce patients' exposure to antibiotics in intensive care units (PRORATA trial): a multicentre randomised controlled trial. Lancet 375: 463-474.

13. Rodrigo Octavio Deliberato, Alexandre R. Marra, Paula Rodrigues Sanches (2013) Clinical and economic impact of procalcitonin to shorten antimicrobial therapy in septic patients with proven bacterial infection in an intensive care setting. Diagn Microbiol Infec Dis 76: 266-271.

14. Marcel Hochreiter, Thomas Köhler, Anna Maria Schweiger (2009) Procalcitonin to guide duration of antibiotic therapy in intensive care patients: a randomized prospective controlled trial. Critical Care 13: 83-89.

15. Evelien de Jong, Jos A van Oers, Albertus Beishuizen (2016) Efficacy and safety of procalcitonin guidance in reducing the duration of antibiotic treatment in critically ill patients: a randomised, controlled, open-label trial. Lancet Infect Dis 16: 819-827. [Crossref]

16. Nathalie Layios, Bernard Lambermont, Jean-Luc Canivet (2012) Procalcitonin usefulness for the initiation of antibiotic treatment in intensive care unit patients. Crit Care Med 40: 2304-2309.
17. Baohua, LI Haifeng, LEI Yu (2013) Clinical significance of dynamic monitoring of procalcitonin in guiding the use of antibiotics in patients with sepsis in ICU. Chin Crit Care Med 25: 690-693. [Crossref]

18. Atabak Najafi, Ali Khodadadian, Mehdi Sanatkar (2015) The Comparison of Procalcitonin Guidance Administer Antibiotics with Empiric Antibiotic Therapy in Critically Ill Patients Admitted in Intensive Care Unit. Acta Medica Iranica 53: 562-567.

19. Vandack Nobre, Stephan Harbarth, Jean-Daniel Graf (2008) Use of Procalcitonin to Shorten Antibiotic Treatment Duration in Septic Patients: A Randomized Trial. AMJ RESP CRIT CARE 177: 498-505. [Crossref]

20. Schroeder S, Hochreiter, Koehler T (2009) Procalcitonin (PCT)-guided algorithm reduces Length of antibiotic treatment in surgical intensive care patients with severe sepsis: results of prospective randomized study. Langenbecks Arch Surg 394: 221-226.

21. Yahya Shehabi, Martin Sterba, Peter Maxwell Garrett (2014) Procalcitonin Algorithm in Critically Ill Adults with Undifferentiated Infection or Suspected Sepsis: A Randomized Controlled Trial. AM J RESP CRIT CARE 190: 1102-1110.

22. Petr Svoboda, Ilona Kantoroua, Peter Scheer (2007) Can Procalcitonin help us in timing of re-intervention in septic patients:after multiple trauma or major surgery? Hepato-Gastroenterol 54: 359-363. [Crossref]

23. Schuetz P, Muller B, Christ-Crain M, Stolz D, Tamm M, et al. (2012) Procalcitonin to initiate or discontinue antibiotics in acute respiratory tract infections. Cochrane Database Syst Rev 8: 1297-1371. [Crossref]

24. Emilio Maseda, Alejandro Suarez-de-la-Rica, Victor Anillo (2014) Procalcitoninguided therapy may reduce length of antibiotic treatment in intensive care unit patient with secondary peritonitis: A multicenter retrospective study. Journal of Critical Care 30: 537-542. [Crossref]

25. JA Carr (2015) Procalcitonin-guided antibiotic therapy for septic patients in the surgical intensive care unit. Journal of Intensive Care 3: 1-8.

26. A Prkno, C Wacker, FM Brunkhorst (2013) Procalcitonin-guided therapy in intensive care unit patients with severe sepsis and septic shock--a systematic review and metaanalysis. Crit Care 17: 291. [Crossref]

27. JU Jensen, L Hein, B Lundgren (2013) Procalcitonin-guided interventions against infections to increase early appropriate antibiotics and improve survival in the intensive care unit: a randomize trial. Crit Care Med 17: 291. [Crossref]

28. Ahmad I (2009) Hospital and societal costs of antimicrobial-resistant infections in a Chicago teaching hospital: implications for antibiotic stewardship. Clin Infect Dis 49: 1175-1184

29. Christ-Crain M, Jaccard-Stolz D, Bingisser R (2004) Effect of procalcitonin-guided treatment on antibiotic use and outcome in lower respiratory tract infections: Clusterrandomised, single-blinded intervention trial. Lancet 363: 600-607. [Crossref]

30. Schuetz P, Christ-Crain M, Thomann R (2009) ProHOSP Study Group: Effect of procalcitonin-based guidelines vs standard guidelines on antibiotic use in lower respiratory tract infections: The ProHOSP randomized controlled trial. JAMA 302: 1059-1066. [Crossref]

Copyright: (C2018 Yuhan ZHU. This is an open-access article distributed under the terms of the Creative Commons Attribution License, which permits unrestricted use, distribution, and reproduction in any medium, provided the original author and source are credited. 